\title{
La vulneración del derecho fundamental de las víctimas de violencia sexual
}

\author{
Violation of the fundamental rights of victims of sexual violence \\ Violação do direito fundamental das vítimas de violência sexual
}

Artículo recibido en abril 2021

Arbitraje en mayo 2021

Aceptación en junio 2021

Publicación en julio 2021
Karina Natali Crisóstomo Talla

kcrisostomo@ucvvirtual.edu.pe

https://orcid.org/ 0000-0003-0753-557X

Universidad César Vallejo, Lima-Perú
RESUMEN

ABSTRACT

RESUMO
Con frecuencia se mencionan en los medios de comunicación cómo las mujeres son víctimas de violencia sexual. El estudio muestra un análisis de la vulneración del derecho fundamental de las víctimas de violencia. El estudio se llevó a cabo mediante la revisión bibliográfica de artículos científicos de revistas usando motores de búsquedas como Google Académico, además de indexadores o plataformas como en Scielo, Redalyc, Scopus, Eric, entre otras. Como resultado de la investigación se evidenció que las mujeres han sufrido de violencia sexual, causando un gran impacto y desprecio entre las personas y la sociedad. Se concluye que las mujeres con mayor riesgo de sufrir violencia sexual son aquellas que dependen económicamente de sus parejas, mujeres con estudios primarios y secundarios, con poca autoestima personal, con miedo e incapaces de denunciar al agresor.

Palabras clave: Violencia sexual; Violación; Libertad; Factores de riesgo

It is frequently mentioned in the media how women are victims of sexual violence. The study shows an analysis of the violation of the fundamental right of victims of violence. The study was carried out through a bibliographic review of scientific journal articles using search engines such as Google Scholar, as well as indexers or platforms such as Scielo, Redalyc, Scopus, Eric, among others. As a result of the research it was evidenced that women have suffered sexual violence, causing a great impact and contempt among people and society. It is concluded that women at greater risk of suffering sexual violence are those who are economically dependent on their partners, women with primary and secondary education, with low personal self-esteem, fearful and unable to denounce the aggressor.

Key words: Sexual violence; Rape; Freedom; Risk factors

A forma como as mulheres são vítimas de violência sexual é freqüentemente mencionada na mídia. $\mathrm{O}$ estudo mostra uma análise da violação do direito fundamental das vítimas de violência. $\mathrm{O}$ estudo foi realizado através de uma revisão bibliográfica de artigos de revistas científicas utilizando motores de busca como o Google Scholar, assim como indexadores ou plataformas como Scielo, Redalyc, Scopus, Eric, entre outros. Como resultado da pesquisa, tornou-se evidente que as mulheres sofreram violência sexual, causando grande impacto e desprezo entre as pessoas e a sociedade. Conclui-se que as mulheres com maior risco de sofrer violência sexual são aquelas que dependem economicamente de seus parceiros, mulheres com educação primária e secundária, com baixa auto-estima pessoal, com medo e incapazes de denunciar o agressor.

Palavras-chave: Violência sexual; Violação; Liberdade; Fatores de risco 


\section{INTRODUCCIÓN}

La violencia sexual contra las mujeres es un flagelo tanto a nivel internacional, nacional y local; cuya violencia genera un gran impacto y rechazo entre las personas que habitan en cada país o sociedad en la cual se ha visto que la población se ha levantado saliendo a las calles para que sus voces sean escuchadas y sobre todo que los agresores sean identificados, capturados y sancionados, cuya finalidad de dicha manifestación es que las victimas puedan encontrar justicia a la violencia perpetrada en aquellas mujeres. Las víctimas de este tipo de violencia refieren que los agresores se comportan o tienen una actitud machista, autoritario y violento debido a que estas personas (agresor) han crecido en un entorno al consumo del alcohol, otros factores se deben a que han tenido una infancia donde su padre maltrataba a su madre, es decir, en un hogar de violencia doméstica, siendo testigos de maltrato físico a la mujer. Así mismo, agresores con aptitud machista por el solo hecho de ser del género masculino se sienten con la autoridad de poder maltratar física como sexualmente a sus parejas.

En ese sentido, el análisis a nivel internacional como en España con Rodriguez et al., 2014), Estados Unidos con Krebs en el 2011, Uganda con Karamagi en el 2006 y Nicaragua con Ellsberg en el 2001, se encontraron hallazgos similares sobre violencia sexual, lo que se confirma que, a pesar de la diversidad de culturas, costumbres en cada país o sociedad se evidencia que el maltrato físico se conduce hacia la violencia sexual.

En Perú estudios hallados por Alarcón y Ortiz, (2017); Blitchtein y Reyes, (2012); Castro et al., (2017); Flake; (2005) reportaron que las víctimas de violencia sexual manifestaron que su pareja (agresor) con frecuencia consumen alcohol, siendo en dicho estado es que sufren de estos maltratos físicos y sexual. Uno de los factores del alcoholismo se debe a que no cuentan con un trabajo estable, suelen tener trabajos eventuales, esporádicos y poco remunerados. A partir de ello los autores concluyeron que aquellas mujeres con parejas que consumen alcohol hasta el punto de la embriaguez a menudo tienen más probabilidades de experimentar violencia sexual. Este comportamiento agresivo, reprochable y abominable de los agresores generan un total rechazo y repudio por parte de la sociedad o dentro de un estado de derecho. En la cual las personas, llámense familiares de las victimas salen a las calles en voz de protesta a defender los derechos de las mujeres que han sido víctimas de violencia, buscando la máxima sanción para los agresores e incluso pidiendo cadena perpetua, para que otras mujeres no sean víctimas de aquellos; y sus víctimas por los menos se sientan protegidas por el estado (operadores de justicia) de no dejarlos libres por la ciudad o peor aún que se puedan acercar para amenazarlas y reiterar el abuso cometido. Ante esta realidad en que viven las mujeres 
de violencia sexual y física se considera que se debe poner énfasis en analizar cuáles son los factores de riesgos a la cual están propensas, identificar cuáles son los indicios o características de los agresores, en la cual las mujeres deben tener cuidado, desde el mínimo de violencia, comenzando desde la violencia verbal.

El propósito del manuscrito radica en analizar la vulneración del derecho fundamental de las víctimas de violencia sexual. La importancia del estudio está esbozada en un análisis para evidenciar los daños que tienen las victimas tras haber sufrido alguna agresión de violencia sexual, con la finalidad de mantener a esta población de mujeres a estar atenta al comportamiento o actos de sus parejas, los cuales en algunos momentos suelen ser características de violencia y alertarlas que dicho comportamiento puede causarle un daño moral, físico, irreparable y hasta mortal. Así mismo se considera que esta problemática que aquejan a estas mujeres víctimas de violencia sexual es de suma importancia; por cuanto en la actualidad las víctimas se centran en la persecución de su agresor a que estos paguen con la mayor pena privativa de libertad, que sean sancionados hasta con cadena perpetua y sobre todo que paguen una fuerte suma de dinero por los daños ocasionados los cuales son irreparables, porque no existe un monto de dinero que le pueda devolver su tranquilidad, su paz moral, psicológico. Asimismo, en sociedad se pernotan que los operadores de justicia centran su sanción en contra los agresores en una reparación económica dejando de lado la reparación psíquica, moral, subjetiva, psicológica de la víctima de violencia sexual.

\section{METODOLOGÍA}

El estudio se llevó a cabo mediante la revisión bibliográfica de artículos científicos de revistas usando motores de búsquedas como Google Académico, además de indexadores o plataformas como en Scielo, Redalyc, Scopus, Eric, entre otras. El resultado de la búsqueda fue mediante la dimensión trabajada acerca de la violencia sexual, para ampliar la búsqueda se usaron términos como violación, agresores sexuales, violencia sexual en Perú, victimas sexuales. La obtención de datos refiere al análisis de 17 artículos de investigación de los cuales se eligió 15 artículos en función a la investigación que son relevantes sobre las categorías de estudio, 19 sobre el marco teórico (Figura 1), además también se analizaron el marco teórico para sustentar y discutir la dimensión y variables abordadas. El método de estudio empleado fue el inductivo. 


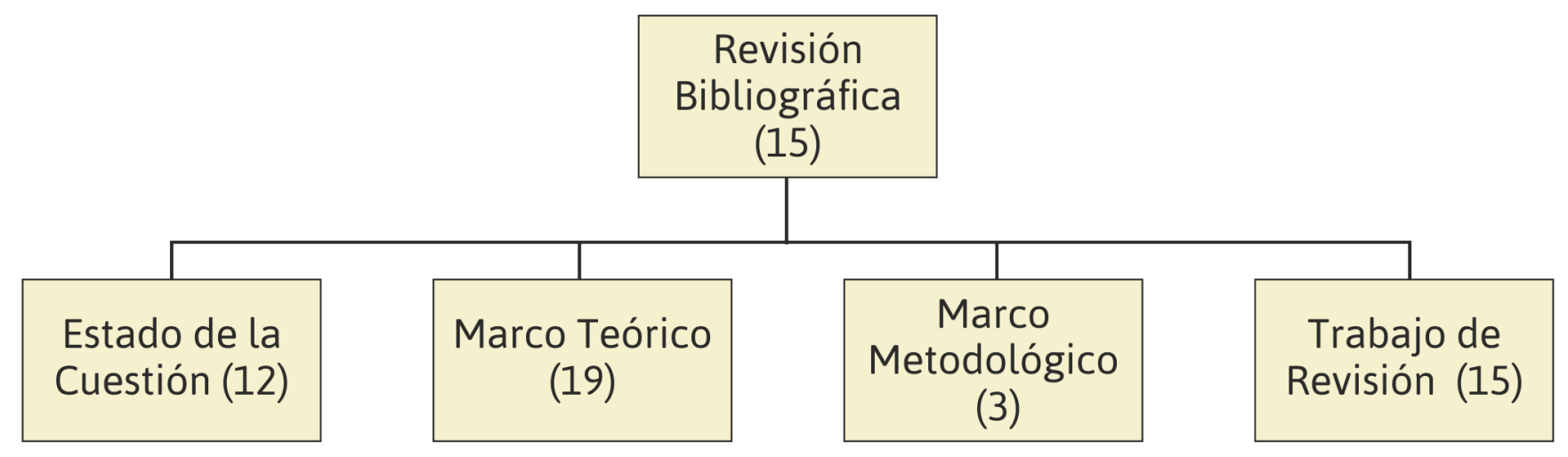

Figura 1. Resultado de la búsqueda bibliográfica.

\section{Búsqueda de estudios}

Se efectuó una investigación manual esgrimiendo las referencias de los estudios primarios y secundarios que se encontraron en la búsqueda electrónica, siendo factible ubicarlos en la base de datos de Scielo, entre otras, que cuentan en la plataforma virtual de la Universidad Cesar Vallejo y paginas académicas.

\section{DESARROLLO Y DISCUSIÓN}

A continuación, se presenta un análisis de los artículos relacionados con la violencia sexual en Perú y en otros países.

Se inicia con el estudio de Enríquez et al., (2020) quienes realizaron una investigación en Perú con una muestra de 21414 con un grupo social con edad comprendida entre 15 a 49 años. El método utilizado fue en participantes. Los resultados mostraron que en la información efectuada por ENDES de 2017 señalaron haber tenido una pareja y que manifestaron afirmativamente a la sección de violencia. De ello, los resultados de la muestra arrojaron que el $6.3 \%$ sufrió maltrato sexual por parte de su pareja en el último año, además el $29.5 \%$ sufrió de maltratos físicos, mientras que el $43.6 \%$ mostró tener precedentes de maltratos del padre hacia la madre. Por otro lado, un $15 \%$ de la muestra mostró haber sido amenazada económicamente y $73.1 \%$ dijo que los compañeros se embriagaban ciertas veces. En base a ello, siguiendo una perspectiva ecológica, el riesgo de experimentar violencia sexual aumenta al haber presenciado casos de maltratos del padre hacia la mamá y haber sido víctima de maltratos físicos por parte de su 
progenitor. La relevancia de este estudio radicó en identificar las actividades preventivas a nivel primario ante casos de violencia sexual. Ello a causa de que la comunicación entre las parejas resulta ser de gran relevancia como medio protector ante tales casos. Por otro lado, tales actividades primarias también resultan importante realizarlas en parejas que presentan diversos factores riesgosos, tales como ser convivientes, pertenecer a sectores de bajos ingresos, vivir en sectores rurales, o aquellos que hayan manifestado algún tipo de violencia física por parte de algún compañero.

Seguidamente, en el estudio presentado por Sosa y Menkes (2020) corresponde a una investigación original, realizada en México que tuvo como muestra a 29878 mujeres de 15 a 29 años. El método que utilizaron fue la encuesta efectuada por entidades u organismos que recaban información respecto a la violencia de género y la convivencia en los domicilios. Los resultados mostraron que el 78.1 \% de la muestra son jóvenes de 15 a 29 años. De tales mujeres, "el $7.5 \%$ tienen descendencia y entre éstas, $28.7 \%$ tuvo su primogénito entre los 13 a 17 años de edad, $51.8 \%$ entre los 18 a 22 años de edad, $15.3 \%$ entre los 23 a 26 años y $2.1 \%$ entre los 27 y 29 años" (Sosa, 2015). El análisis realizado en tal estudio mostró que ciertas causas se deben al estrato económico y la educación las cuales tienen una relación estadísticamente significativa con respecto al maltratos en la relación de novio. Así mismo, el multivariado estudio señala que algunos componentes del maltrato sexual a señoras jóvenes son el tener hijos, nula autonomía de la mujer y escasa libertad personal dentro de una relación de noviazgo. Así mismo, pertenecer a un estatus social económico muy ruin también presenta una relación significativa. Por otro lado, el tener una relación toxica desde el inicio y que aumente progresivamente durante la duración de esta. Tales problemas son resueltos en su mayoría por el género masculino de forma imponente. Como conclusión se evidencia que la violación sexual está fuertemente relacionada con otros tipos de violencia, así como por desigualdades de género. La relevancia de este estudio se destaca en destruir toda creencia, costumbre y culturas sobre violencia sexual.

Se continua con Lozano et al., (2017) quienes han descrito en su investigación en España, cuya población de estudio fue 61 mujeres de edades entre 16 y 35 años. El método que utilizaron fue la entrevista semiestructurada. Mediante las entrevistas se lograron recaudar datos sociodemográficos, algunas características principales de la agresión, como la edad frecuente de agresión y la constancia del evento. Respecto a ello, los principales resultados mostraron diferentes variables del Trastorno de Estrés Postraumático encontrados en todos los subgrupos de mujeres que sufrieron violencia sexual, en un índice más elevado. Esto indicaría que síntomas del TEP son más claro en estos tipos de violencia. Como conclusión, es posible decir 
que la dimensión de la población de estudio y los grupos generados en ella, hace que se deba prestar especial atención a ello y con prudencia. Así mismo, la importancia del informe está en la aportación de información hacia temas de violencia contra las mujeres y en el camino de mejorar la prevención primaria y secundaria.

Para completar el análisis, Ferrel et al., (2020) señalan en su investigación realizada en Colombia, la cual tuvo como muestra a mujeres desplazadas que hayan sido víctimas de violencia sexual entre los 20 y 55 años. El método utilizado fue descriptivo correlacional. El resultado de este método demostró que el $80 \%$ de estas mujeres se encontraban en niveles muy altos de estrés postraumáticos, a diferencia del grupo de mujeres desplazadas que no han sido víctimas. En tanto, un 40\% de mujeres de la muestra presentaron tener una forma de vida inadecuada, a diferencia del otro grupo de mujeres no víctimas. Tal efecto muestra el impacto negativo que tiene el trastorno de estrés postraumático (TEP), y más en aquellas mujeres que han sido doblemente victimizadas en comparación a aquellas que sufrieron forzosamente de desplazamiento. Ello ha demostrado que dicho trastorno afecta el estilo de vida y la salud de la población estudiada. Sin embargo, no se ha logrado comprobar correlación fuerte entre el estilo de vida y el TEP. Con ello, es posible evidenciar que la importancia de este estudio fue mediante la identificación de una relación existente entre la presencia de TEP y la forma de vida de la persona afectada.

Para Fernández, Quiñones y Prado (2019) la cual tuvo como población de estudio al menos 50 mujeres víctimas de violencia a quienes fue dirigida la entrevista. Ante esto, el método de estudio utilizado fue el de las encuestas individuales y los instrumentos, que sirvieron de guía para las entrevistas, además de formar escalas del perfil del agresor y una ficha de valoración sobre la violencia en la pareja. Ante la descripción del perfil del agresor, los resultados demostraron que la mitad de las mujeres estudiadas ubicaron a su agresor en un nivel medio; mientras que la otra mitad, lo ubicaron en un nivel bajo. Por otro lado, se encontró que los agresores intentaban controlar a sus víctimas (sus parejas) mediante amenazas e intimidación o, como en otros casos, a través de actos humillantes o de crueldad hacia las víctimas (Fernández, ob. cit. 2019). En conclusión, la información de este estudio presenta un aporte muy significativo para mejorar la sensibilización del personal sanitario y de los profesionales encargados de detectar el perfil del agresor en situaciones violencia en pareja, y registrar tales actos como comportamientos frecuentes de maltrato. Toda esta información recolectada sería de gran aporte para las instancias del sistema legal con la finalidad de que estos puedan comprender mejor las personalidades, conductas y actitudes del agresor. Esto permitiría tomar mejores acciones de 
prevención y el ofrecimiento de mejores servicios especializados ante casos psicopatológicos. La relevancia del estudio, entonces, recaería en su determinación para relacionar el perfil del agresor y los casos de violencia hacia la pareja.

Otro estudio analizado fue el presentado por Borbor (2019) correspondiente a una investigación originaria en Ecuador que tuvo como análisis a mujeres pertenecientes a la Provincia de Santa Elena. El método que utilizaron fue el método mixto donde se ejecutó varias preguntas que fueron realizadas a diferentes personas de dicha provincia, con la intención de saber si las mujeres conocen sus derechos, si dogmatizan que todavía se vive en un medio donde la mujer se somete a las decisiones del hombre o que se creen y siente culpables por la violencia sexual que han sufrido a causa de su forma de vestir, pensar o actuar, por último que piensan sobre la violencia sexual y el conocimiento sobre cuantas violaciones, acosos y abusos se han llevado a cabo desde el año 2015. Resultados Los resultados obtenidos dieron a conocer que las mujeres ocultan la violencia sexual por temor y necesitan de alguien de confianza para poder hablar, los que comente el delito de violación sexual son personas cercanas a las víctimas mayormente, las victimas en algunos casos viven con una criatura producto de una violación que tuvieron que aceptar el hecho de aun repudiando el acto, son madres, concluye se deben fomentar en esta sociedad provincial una campaña de concientización del hombre con relación al respeto hacia la mujer, la mujer no es menos que el hombre, ya que se está en igualdad de condición, igualdad de derecho, por lo tanto, el Estado es el responsable de garantizar todos los derechos establecidos constitucionalmente. La importancia y relevancia de este estudio fue documentar el conocimiento de las mujeres sobre la violencia sexual.

Para continuar con el análisis, Lira et al., (2017) muestran en su estudio desarrollado en Chile que tuvo como muestra 174 hombre sentenciados por el delito de violencia sexual. El método que se usó fue a través de una muestra constituida mediante un proceso no probabilístico, el cual correspondía en registrar a todas las personas condenadas por violencia sexual en el periodo de abril de 2014 en el establecimiento Penitenciario de Chile. En la muestra se incluyeron un total de 174 personas recluidas, mientras que se lograron incluir 208 condenas de tipo. Del total de la muestra, los autores mencionan que el $42,3 \%$ presenta una o dos penas privativas de libertad por el delito de violación sexual (el 9,1\% de ellas recae a personas con dos censuras por el mismo delito). Mientras que, se aprecia un $21,6 \%$ de hombres censurados por uno o más delitos de Violencia sexual mayor de 14 años" (de los cuales 5,17\% cuenta entre dos a siete censuras por el mismo delito). De acuerdo con ello, se pueden describir las características que destacan en el grupo de agresores sexuales estudiado: "hombres, mayormente solteros de 40 
a 49 años de edad, así como hombres de 30 a 39 años de edad que cuentan con niveles de estudios medios que habitan en comunas (seguidos por el grupo de 30-39 años), escolaridad en niveles medios, residentes en comunidades de más de 200.000 habitantes, con una minoría de peligrosidad en su actuar delictivo, quienes mayormente vienen cumpliendo condenas por el delito de violencia sexual. (Lira ob. cit. 2017). La relevancia de este estudio radicó en encontrar características distintas a las usualmente estudiadas.

En Pinto-Cortez, y Guerra (2019) corresponde a otro estudio presentado en Chile. El método que utilizaron para obtener a muestra de estudio fueron los provenientes de la ENP. El cuestionario que se usó evaluaba diferentes formas de violencia que afectan durante la infancia, las cuales se agruparon, de manera pertinente para el estudio, en seis secciones o módulos: victimización por delitos comunes, victimización por cuidadores, victimización por hermanos, victimización sexual, victimización indirecta y victimización electrónica. De ello, los resultados fueron que el $26,4 \%$ de los integrantes de la muestra mostraron haber sufrido al menos de un tipo de victimización sexual a lo largo de toda su vida. Así mismo, se encontró que la proporción de mujeres que menciona haber sufrido al menos algún tipo de victimización sexual durante toda su vida es mayor al de los varones en alrededor de 10\%; mientras que un 16,7\% reportó haber sufrido al menos un tipo de victimización sexual en los últimos 12 meses, siendo en su mayoría las mujeres quienes lo reportaron. Como conclusión, es posible decir que, si bien el riesgo de sufrir de violencia sexual perjudica tanto a hombres como a mujeres, son estas últimas quienes tienen mayor probabilidad de padecerla alguna vez en su vida. Por otro lado, otra conclusión importante a partir del estudio fue que los niños migrantes, adolescentes entre 15 y 18 años, quienes presentan alguna discapacidad, quienes estudian en colegios subvencionados $y$ municipales, y quienes viven en zonas diferentes a la Metropolitana sufren un mayor riesgo de sufrir algún tipo de violencia sexual. La relevancia del estudio fue evidenciar la vigencia de casos de victimización sexual y otras características asociadas, dentro de una muestra de niños y adolescentes.

La agresión sexual de pareja se desarrolla a través de las distintas causas que existen en el tipo ecológico (Heise, 1998; OMS, 2002). Entre las causas personales se encontró que las víctimas de violencia familiar son más propensas a experimentar violencia sexual reciente. Al respecto, se encontraron resultados equivalentes en España con Rodríguez en el 2014, EE.UU. con Krebs en el 2011, Uganda con Karamagi en el 2006 y Nicaragua con Ellsberg en el 2001, con lo que se verifica que, a pesar de las diferentes culturas, la agresión física suele estar anexada por casos de violencia sexual. Respecto a los factores económicos, "un análisis chileno indicó un 
48 \% de violencia se debe al factor económico (Barría-Muñoz y Macchiavello-Rodríguez, 2012), “confrontado con un $\mathbf{2 9 . 3 \% ~ e n ~ j o ́ v e n e s ~ q u e ~ h a b i t a n ~ e n ~ l a ~ c i u d a d ~ d e ~ M e ́ x i c o ~ ( C a s i q u e , ~ 2 0 1 0 ) . ~ L o s ~}$ resultados del análisis reflejan que un 15 \% que experimentó amenazas económicas.

Por otro lado, los resultados validan que las mujeres propensas a experimentar violencia sexual recientes son las que cuentan con parejas que beben licor de manera frecuente. Este hecho se reporta en otros estudios similares tanto en como a nivel internacional. En nuestra sociedad, el consumo de alcohol está ligado al sexo masculino y al rol social que se le asigna (Castro et al., 2017). En el presente estudio, los datos corroboran que el $70 \%$ de las mujeres informan que su pareja bebe licor en cualquier momento. Esta información es alarmante, puesto que el consumo de bebidas alcohólicas, desde antaños ha estado conexo con los maltratos físicos y sexuales (Puente-Martínez et al., 2016).

Del análisis elaborado por ENDIREH (2011), se puede evidencia que los casos de violencia sexual dentro de relaciones de noviazgo están fuertemente ligada a las desigualdades de género previamente instauradas y aprendidas en la sociedad que rodea a la pareja, y no exclusivamente por casos de desigualdad dentro del noviazgo. Los resultados de este estudio también señalan que las mujeres con hijos son quienes tienen mayor probabilidad de ser víctima de una violación sexual. Ello, en conjunto con estudios previos, podría estar sugiriendo ciertos vínculos existentes entre las experiencias reproductivas, sexuales con la violencia sexual.

Por otro lado, en base a los resultados, no se han podido identificar diferencias significativas entre los grupos de estudio; sin embargo, sí se ha podido encontrar diferentes indicadores de casos de trastornos de estrés postraumático en mujeres que han sufrido de agresión sexual. Ello se debe a que, en los diferentes subgrupos de estudio, un número grande de mujeres alcanzaban niveles preocupantes de TEP, lo que permitió asociar esta sintomatología con la agresión sexual. Luego, también es posible decir que de los resultados obtenidos se ha podido evidenciar que las mujeres que han sufrido de violencia sexual, en su mayoría se han encontrado en niveles malos, inadecuados e irregulares de estilos de vida. Con esto, se hace claro el impacto negativo no solo en la salud mental o física, sino también en la calidad o estilo de vida de la persona agredida, llegando a impactar incluso en el proceso de recuperación de la víctima y su reinserción en la sociedad.

De acuerdo con Echeburúa et al., (2016) el absoluto de las mujeres que sufrieron de violencia sexual podía catalogarse en un nivel crónico de afectación pues aún después de meses de ocurrido el evento, estas seguían presentando síntomas. Así pues, estas mujeres pueden 
presentar estos síntomas de "volver a vivir" tal evento traumático hasta 10 años después de su ocurrencia, lo que perjudicaría aún más la calidad y el modo de vida de la víctima.

De los resultados también se encontró que las desigualdades de género determinadas en la sociedad pueden beneficiar la ocurrencia de agresiones sexuales, apoyado en los estereotipos y la discriminación (González, 2007). En concordancia con ello, Gonzáles y Fernández (2010) encontraron que las víctimas de tal violencia señalan como causas principales los celos del agresor y su frecuente consumo excesivo de alcohol. Estas acciones, que son características como "comunes" en el género masculino, facilita la desinhibición de la pareja agresora que incita el manejo del enojo y la frustración a través de la violencia física y sexual.

Los resultados mostrados por el autor dan a conocer cómo las mujeres de Santa Elena también son víctimas de violencia sexual a pesar de ser una provincia pequeña. En esta provincia se está viviendo una época peligrosa, por cuanto ni las mujeres adultas se libran de alguna violencia, las leyes no son respetadas, los administradores de justicia no colaboran con los que más lo necesitan. Asimismo, las mujeres que habitan en esta provincia sin importar la edad que tengan son víctimas de violencia sexual muchas veces por su forma de vestir, sin embargo, el problema es el pensamiento de ciertos hombres machistas y de la sociedad machista que aún existe.

En relación a lo señalado en los diferentes estudios internacionales (Noguerol, 2005), el 98\% de los agresores corresponden al sexo masculino. Al respecto y a diferencia de la literatura revisada, los resultados muestran que mayormente los agresores corresponden a hombres solteros en casi un 50\%, en comparación a los hombres casados que están alrededor del 30\%, aunque no era conocido si los hombres de estado civil soltero mantenían alguna relación con las agraviadas, de acuerdo con Ruiz, y Crespo (2010). Este hallazgo resultó ser similar al encontrado previamente por Muñoz en Chile (2013). No obstante, según lo encontrado por Arcila, no es correcto precisar, respecto a los niveles educacionales encontrados, que la mayor parte de la muestra presenta bajos índices de escolaridad, ello debido a que el 63,8\% de esta demostró alcanzar a cursar la escolaridad media, llegando incluso un $11 \%$ a alcanzar niveles superiores de educación. Tales resultados evidenciarían una mayor concentración de individuos en niveles de educación medios, técnicos y superiores.

Los resultados obtenidos son preocupantes debido a que cerca del $26 \%$ de las participantes reportó al menos un tipo de agresión sexual en toda su vida mientras que alrededor del 17\% menciona haber sido víctima de por lo menos un tipo de violencia sexual en los últimos meses antes del estudio. Con tales datos es posible observar la intensidad del problema, su prevalencia 
y vigencia. Ante esto surge la necesidad de realizar evaluaciones continuas con el fin de identificar estos casos y tipos de violencias, ello con el fin de generar acciones de prevención o intervención en el caso de violencia sexual (Saywitz, Mannarino, Berliner, y Cohen, 2000). Así mismo, los resultados señalan que los adolescentes mayores tienen mayor probabilidad de sufrir abusos sexuales, lo que es coherente con la teoría de la victimología del desarrollo (Finkelhor, 2007), la cual sostiene que, a medida que la edad aumenta, los adolescentes van acumulando un mayor número de victimizaciones en su vida, lo que implica, que este efecto acumulativo ocasione daños a nivel neurobiológico, cognitivo y afectivo (Ford, Wasser, y Connor, 2011).

\section{CONCLUSIONES}

Respecto a la violencia sexual, se ha evidenciado que las causas que incrementan la probabilidad de agresión sexual se deben a que las victimas desde edades temprana fueron testigos cómo sus madres también fueron víctimas de violencia física, sexual, psicológica, entre otras. Asimismo, los padres de las victimas desde edades temprana edad ejercían violencia física sobre la víctima, relación hija-padre.

Respecto a la agresión sexual, dentro de una relación está ligada con las desigualdades de género, en la cual el hombre cuenta con la errada idea que la mujer se encuentra en un estado de inferioridad a él. Y, por tanto, las decisiones las toma el varón dejando de lado a la mujer o simplemente no las toma en cuenta, el hombre machista siempre cree tener la razón y por último se debe hacer lo que él manda y ordena.

Las mujeres que han sido agredidas mediante agresiones sexuales y que son de mayoría de edad son quienes tienden a tener más síntomas de trastorno de estrés postraumático, debido a que tienden a recordar el suceso dio a día lo que le imposibilita seguir con su vida diaria, es decir tienden a la re-victimización.

El efecto negativo del trastorno de estrés postraumático se mantiene más tiempo en mujeres que han sufrido doblemente de una agresión, ya sea sexual o por desplazamiento. Los efectos negativos se deben a la pobreza en la que se encuentran que le imposibilita seguir un tratamiento psicológico, o simplemente a la marginación a la que se debe enfrentar en la sociedad.

Existe relación entre el perfil del agresor con la violencia de la pareja, una de las características de los agresores es que suelen ser celosos o posesivos, o que al sentirse humillados por la ruptura de la relación tiende a que su autoestima baje, asimismo el excesivo consumo de alcohol o 
drogas. Tienden a comportarse de una manera desafiante y cruel sin temor a las consecuencias físicas, punitivas de su conducta.

El hombre machista cree que es más que la mujer y que en la actualidad no estamos en igual de condiciones, es por ello por lo que tienden a perpetrar estas violencias sexuales, físicas, psicológicas, emocionales, lo que les conlleva a estos actos. El hombre debe entender que las mujeres nos encontramos en equivalencia de condiciones e igualdad de derechos que son reconocidos por todas las instituciones del Estado y debe ser garantizado por este.

Los ofensores sexuales mayoritariamente son solteros que cuentan con niveles de estudios incompletos, escolaridad en niveles medios, residentes en comunas rurales y con un comportamiento delictivo peligroso.

Las mujeres que no cuentan con estudios concluidos son las más propensas de violación sexual a diferencia de las mujeres que sí cuentan con estudios completos o superiores, en el sentido que estas mujeres no permiten ser violentadas por el solo hecho de que saben que se encuentran en un estado de derecho.

\section{REFERENCIAS}

Alarcón, L., y Ortiz, J. (2017). Qué factores se asocian a la vio- lencia psicológica doméstica? Análisis del último decenio de la ENDES. Casus Revista de investigación y casos en salud, 2(1), 11-27. Recuperado de http://casus.ucss.edu. $\mathrm{pe} /$ index.php/casus/article/view/28/12

Arcila Rivera, A., Castaño Ramírez, M., Osorio Montoya, D., \& Quiroz, G. (2013). Caracterización sociodemográfica, del desarrollo psicosexual y del delito en hombres condenados por delitos sexuales recluidos en el centro penitenciario de Manizales. Revista Colombiana de Psiquiatría, 42(1) 51- 62. http:// dx.doi.org/10.1016/S0034-7450(14)60086-X

Barría-Muñoz, J. R., y Macchiavello-Rodríguez, A. (2012). Anatomía de los hombres que ejercen violencia hacia sus parejas: primer levantamiento de datos para el diseño de un perfil. Psicologia.com [Internet], 16(19), 1-29. Recuperado de https://www.academia. edu/27419754/Anatomia_de-los_hombres_ que_ejercen_violencia_hacia_sus_parejas
Blitchtein, D., y Reyes, E. (2012). Factores asociados a vio- lencia física reciente de pareja hacia la mujer en el Perú, 2004-2007. Revista Peruana de Medicina Experimental y Salud Pública, 29(1), 35-43. Recuperado de http://www. scielo.org.pe/pdf/rins/v29n1/a06v29n1.pdf

Borbor, G. (2018). La violencia sexual en mujeres de la peninsula de Santa Elena (Vol. 2, Issue 1). https://orcid.org/0000-0002-0375-8816

Castro, R. J., Cerellino, L. P., \& Rivera, R. (2017). Risk factors of violence against women in Peru. Journal of family violence,32(8), 807-815. doi: https://doi.org/10.1007/ s10896-017-9929-0

Casique, I. (2010). Factores de empoderamiento y protección de las mujeres contra la violencia. Revista mexicana de so- ciología, 72(1), 37-71. Recuperado de http://www.scielo. org.mx/pdf/ rms/v72n1/v72n1a2.pdf

Cortez, C., y Guerra, C. (2019). Victimización sexual de niños, niñas y adolescentes chilenos:prevalencia y características 
asociadas. Articulo. https://scielo.conicyt.

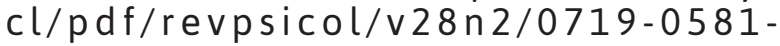
revpsicol-28-2-00020.pdf

Echeburúa, E., y Amor, P. (2016). Hombres violentos contra la pareja: ¿tienen un trastorno mental y requieren tratamiento psicológico? Terapia psicológica, 34(1), 31-40. Recuperado de https://scielo.conicyt.cl/pdf/ terpsicol/v34n1/ art04.pdf

Enríquez, Y., Ortiz, Y., Ortiz, K., y Díaz, G. (2020). Análisis ecológico de la violencia sexual de pareja en mujeres peruanas. Acta Colombiana de Psicología, 23(1), 272-286. https://doi. org/10.14718/ACP.2020.23.1.13

ENDIREH, (2011). La Encuesta Nacional sobre la Dinámica de las Relaciones en los Hogares, INEGI, México

Ferrel, F., Ferrel, L., Caañas,., Barros, M C., y Yañez, H. (2020). Estilo de vida y estrés postraumático en mujeres desplazadas víctimas de abuso sexual en Santa Marta, Colombia. (2020). ARTICULO. https://www.scielosp.org/article/ rcsp/2020.v46n1/e1363/

Ellsberg, M. C., Winkvist, A., Peña, R., y Stenlund, H. (2001). Women's strategic responses to violence in Nicaragua. Journal of Epidemiology \& Community Health, 55(8), 547- 555. doi: http://dx.doi.org/10.1136/jech.55.8.547

Fernández, C., Quiñones, M., y Prado, J. (2019). PERFIL DEL AGRESOR Y VIOLENCIA EN MUJERES DE UNA ZONA PERIURBANA HUÁNUCO, PERÚ. ARTICULO. http://rus.ucf. edu.

Finkelhor, D. (2007). Developmental victimology. En R. C. Davis, A. J. Luirigio, \& S. Herman (Eds.), Vic- tims of crime (3er ed.) (pp. 9-34). Thousand Oaks, California: Sage Publications

Flake, D.F.(2005). Individual, family, and community risk markers for domestic violence in Peru. Violence against women, 11(3), 353-373. doi: https://doi.org/10.1177/107780120427212

Ford, J. D., Wasser, T., y Connor, D. F. (2011). Identifying and determining the symptom severity associated with polyvictimization among psychiatrically impaired children in the outpatient setting. Child Maltreatment, 16(3), 216-226. https://doi. org/10.1177/1077559511406109

González Balderas, T. (2007). Estilos de afrontamiento del estrés utilizados por mujeres víctimas de violencia doméstica. (Tesis de Licenciatura en Psicología). Cholula: Universidad de Las Américas
Heise, L. L. (1998). Violence against women: An integrated, ecological framework. Violence Against Women, 4(3), 262- 290. doi: https://doi. org/10.1177/1077801298004003002

Karamagi,C.A., Tumwine, J. K., Tylleskar, T., y Heggenhougen, K. (2006). Intimate partner violence aga- inst women in eastern Uganda: implications for HIV pre- vention. BMC public health, 6(1), 284. doi: https://doi. org/10.1186/1471-2458-6-284

Krebs, C., Breiding, M. J., Browne, A., y Warner, T. (2011). The association between different types of intimate part- ner violence experienced by women. Journal of Family Violence, 26(6), 487500. doi: https://doi.org/10.1007/ s10896-0119383-3

Lira, G., Varas, C., Salum, S., y Salum, E. (2017). Caracterización sociodemográfica y criminológica de hombres condenadospor delitos sexuales. Revista Psicologica. https:// scielo.conicyt.cl/pdf/revpsicol/v26n1/07190581-revpsicol-26-01-00078.pdf

Lozano Oyola, J. F., Gómez de Terreros Guardiola, M., Avilés Carvajal, I., y Sepúlveda García de la Torre, A. (2017). Sintomatología del trastorno de estrés postraumático en una muestra de mujeres víctimas de violencia sexual. Cuadernos de Medicina Forense, 23(3-4), 8291.

Muñoz, A. M., Kanda, K., Padilla, N., \& Benavente, D. (2013). Caracterización de abusadores sexuales peritados en el Servicio Médico Legal de la VIII región entre los años 2003 y 2011. Trabajo presentado en la III Convocatoria del Concurso de Inves- tigación Forense Distinción Dr. Carlos Ybar, Santiago, Chile

Noguerol, V. (2005). Agresiones sexuales. Madrid, España: Síntesis

Organización Mundial de la Salud [OMS]. (2002). Informe mundial sobre la violencia y la salud. Washington, D.C.: OMS. Recuperado de https:// www.who.int/vio lence_injury_prevention/ violence/world_report/es/summary_es.pdf

Pinto-Cortez,C.yGuerraVio,C.(2019).Victimización sexual de niños, niñas y adolescentes chilenos: prevalencia y características asociadas. Revista de Psicología, 28(2), 1-12. http://dx.doi. org/10.5354/0719-0581.2019.55658

Puente-Martínez, A., Ubillos-Landa, S., Echeburúa, E., y Páez-Rovira, D. (2016). Factores de riesgo asociados a la violencia sufrida por la mujer en la pareja: una revisión de meta-análisis y estudios recientes. Anales de psicología, 
32(1), 295-306. doi: http://dx.doi.org/10.6018/ analesps.32.1.189161

Rodriguez-Menés, J., Puig, D., y Sobrino, C. (2014). Poly-and distinct-victimization in histories of violence against women. Journal of Family Violence, 29(8), 849-858.

Ruiz, J. I. y Crespo, C. (2010). Perfiles sociodemográficos de sujetos condenados en establecimientos colombianos por agresión sexual a adultos o menores. Recuperado de https://goo.gl/6NkiqJ
Saywitz, K. J., Mannarino, A. P., Berliner, L., y Cohen, J. A. (2000). Treatment of sexually abused children and adolescents. American Psychologist, 55(9), 1040-1049. http://dx.doi. org/10.1037/0003-066X.55.9.1040

Sosa, I., y Menkes, K. (2016). Amarte duele. La violación sexual en las relacionesde noviazgo. Un análisis de sus determinantes sociales. ARTICULO. http://www.scielo.org.mx/pdf/pp/ v22n87/1405-7425-pp-22-87-00043.pdf 